\title{
Use of a Novel Negative-Pressure Tent During Bedside Tracheostomy in COVID-19 Patients
}

Venkatakrishna Rajajee ${ }^{1,2,3^{*}}$ (1) and Craig A. Williamson ${ }^{1,2,3}$

(C) 2020 Springer Science+Business Media, LLC, part of Springer Nature and Neurocritical Care Society

\begin{abstract}
Background: Many COVID-19 patients with neurological manifestations and respiratory failure remain dependent on mechanical ventilation and require tracheostomy, which is an aerosol generating procedure (AGP). The risk of SARS-CoV-2 transmission to healthcare staff during AGPs is well documented, and negative-pressure rooms are often unavailable. Innovative techniques to decrease risk to healthcare providers during AGPs are necessary. Our objective was to demonstrate the feasibility of percutaneous dilatational tracheostomy (PDT) performed using a novel prefabricated low-cost negative-pressure tent (AerosolVE).

Methods: Retrospective review of consecutive PDT procedures performed by neurointensivists on intubated adult patients with COVID-19 using the AerosolVE tent during the pandemic under an innovative clinical care protocol. The AerosolVE negative-pressure tent consists of a clear plastic canopy with slits for hand access attached to a U-shaped base with air vents. Air within the tent is drawn through a high-efficiency particulate air filter and released outside. Preliminary testing during simulated AGPs demonstrated negligible escape of particulate matter beyond the tent. The main outcome measure was successful completion of PDT and bronchoscopy within the AerosolVE tent, without complications.
\end{abstract}

Results: The patients were a 53-year-old man with multifocal ischemic stroke and acute respiratory distress syndrome (ARDS), 53-year-old woman with cerebellar hemorrhage and ARDS, and a 69-year-old man with ARDS. Preprocedure $\mathrm{FiO}_{2}$ requirement was $40-50 \%$ and positive end-expiratory pressure (PEEP) 8-12 $\mathrm{cm} \mathrm{H}_{2} \mathrm{O}$. The tent was successfully positioned around the patient and PDT completed with real-time ultrasound guidance in all 3 patients. Bronchoscopy was performed to confirm tube position and perform pulmonary toilet. No complications occurred.

Conclusions: It is feasible to perform PDT on intubated COVID-19 patients using the AerosolVE negative-pressure tent. This is a promising low-cost device to decrease risk to healthcare providers during AGPs.

Keywords: COVID-19, Tracheostomy, Aerosols, Transmission, Acute respiratory distress syndrome, Mechanical ventilation

\section{Introduction}

As the novel coronavirus disease (COVID-19) pandemic evolves, several patients dependent on mechanical ventilation have required tracheostomy.

\footnotetext{
*Correspondence: vrajajee@yahoo.com

${ }^{1}$ Department of Neurosurgery, University of Michigan, 1500 E. Medical Center Drive, 3552 Taubman Health Care Center, SPC 5338, Ann Arbor, MI 48109, USA

Full list of author information is available at the end of the article
}

Approximately $3.2 \%$ of all confirmed COVID-19 cases in China required invasive mechanical ventilation [1], and $17 \%$ of hospitalized patients in one series required intubation [2]. Tracheostomy is an aerosol generating procedure (AGP) similar to endotracheal intubation, extubation, bronchoscopy, manual bag-mask ventilation, administration of nebulized medications, cardiopulmonary resuscitation, noninvasive positive pressure ventilation (NIPPV) and heated high flow nasal cannula

\section{实 Springer}


(HHFNC). The SARS-CoV-2 virus is highly transmissible, and infection of healthcare workers has been widely documented, particularly with performance of AGPs [3, 4]. In one systematic review, healthcare providers caring for severe acute respiratory syndrome (SARS) patients during the 2002-2004 epidemic who performed tracheostomy were four times more likely to contract the disease compared with those who did not [5]. Recent guidelines on performance of tracheostomy on patients with COVID-19 recommend these procedures be performed in negative-pressure rooms (NPR) $[6,7]$; however, the availability of NPRs is typically limited in regions with high case load or limited resources.

Multiple innovative devices designed to decrease the risk of disease transmission during AGPs performed on COVID-19 patients have been reported [8-19]. The majority of these devices are boxes, drapes or tents that surround the patient's head to contain aerosols [12, 1519], while some devices attempt to generate negative pressure to promote clearance of aerosols from the procedural space $[8-11,13,14]$. More recent studies have called into question the role of these devices in the care of COVID-19 patients [20-22]. Containment boxes, tents and drapes without a negative-pressure system do not decrease-and may even increase-the concentration of SARS-CoV-2 virus-sized particles around the proceduralist [20]. There are also concerns that these devices will increase procedural complexity and failure rates $[21,22]$. It is critical therefore that any new device is validated in its ability to minimize the concentration of SARS-CoV-2 virus-sized particles adjacent to the proceduralist, and that the feasibility of performing the procedure in question under real-world conditions on patients be demonstrated. The latter criterion may be especially important for tracheostomy, which is a sterile procedure that is more complex and requires more procedural space than intubation, particularly if performed with bronchoscopy. Devices that generate negative pressure must demonstrate an air-exchange rate that meets the standards of the Centers for Disease Control and Prevention (CDC) for NPRs [23].

\section{Objective}

To demonstrate the feasibility of percutaneous dilatational tracheostomy (PDT) performed using a novel low-cost negative-pressure tent (AerosolVE).

\section{Setting}

Intensive care units providing care for COVID-19 patients.

\section{Participants}

Intubated adult patients with COVID-19 requiring tracheostomy in April and May 2020.

\section{Design}

The institutional review board (IRB) reviewed the study and waived the need for approval. This is a retrospective review of consecutive PDT procedures performed using the AerosolVE tent on patients with COVID-19. Clinical use of the tent during the pandemic was under an institutional innovative clinical care protocol. Informed consent was obtained from legally authorized representatives for use of the tent, as well as acquisition of photographic images and video.

The AerosolVE tent was developed at the Michigan Center for Integrative Research in Critical Care, University of Michigan, Ann Arbor, MI, in collaboration with FlexSys Inc, Ann Arbor, MI. The tent consists of a clear plastic canopy with multiple slits that allow access to the patient, supported by a foldable frame. The tent is attached to a portable U-shaped base and can be folded back onto the base (Fig. 1). Air within the tent is drawn out through a high-efficiency particulate air (HEPA) filter via negative pressure generated by a vacuum motor. Air within the tent is exchanged over 600 times/h, 50 times the 12 air cycles/h recommended by the CDC [23]. While the clear plastic canopy and HEPA filter are disposable following a procedure, the base may be reused for the same patient or other patients following disinfection with anti-viral wipes appropriate for SARS-CoV-2.

\section{Preliminary Data}

Initial testing was previously performed with healthy volunteers and a particle generator (TSI Inc, Shoreview, $\mathrm{MN}$, USA) to simulate aerosol generation by a COVID19 patient [24]. A condensation particle counter (Model 3007, TSI Inc, Shoreview, MN, USA) was used, which detects particles $0.01 \mu \mathrm{m}$ to $>1 \mu \mathrm{m}$ (the SARS-CoV-2 virus is $0.06-0.14 \mu \mathrm{m})$. The particle content of ambient room air was $100-300$ particles $/ \mathrm{cm}^{3}$. The use of HHFNC $60 \mathrm{~L} / \mathrm{min}$ combined with use of the particle generator generated a mean air particle content (mAPC) inside the tent of $18,867 / \mathrm{cm}^{3}$, while the particle count outside was $139 / \mathrm{cm}^{3}$. Addition of a nebulizer mask with saline solution at $10 \mathrm{~L} / \mathrm{min}$ to HHFNC $60 \mathrm{~L} / \mathrm{min}$, without the particle generator, generated a $\mathrm{mAPC}$ inside the tent of $66,835 / \mathrm{cm}^{3}$, while the count was $338 / \mathrm{cm}^{3}$ outside. The use of continuous positive airway pressure (CPAP) in association with the particle generator resulted in a mAPC inside the tent of $27,802 / \mathrm{cm}^{3}$, and $179 / \mathrm{cm}^{3}$ outside. 


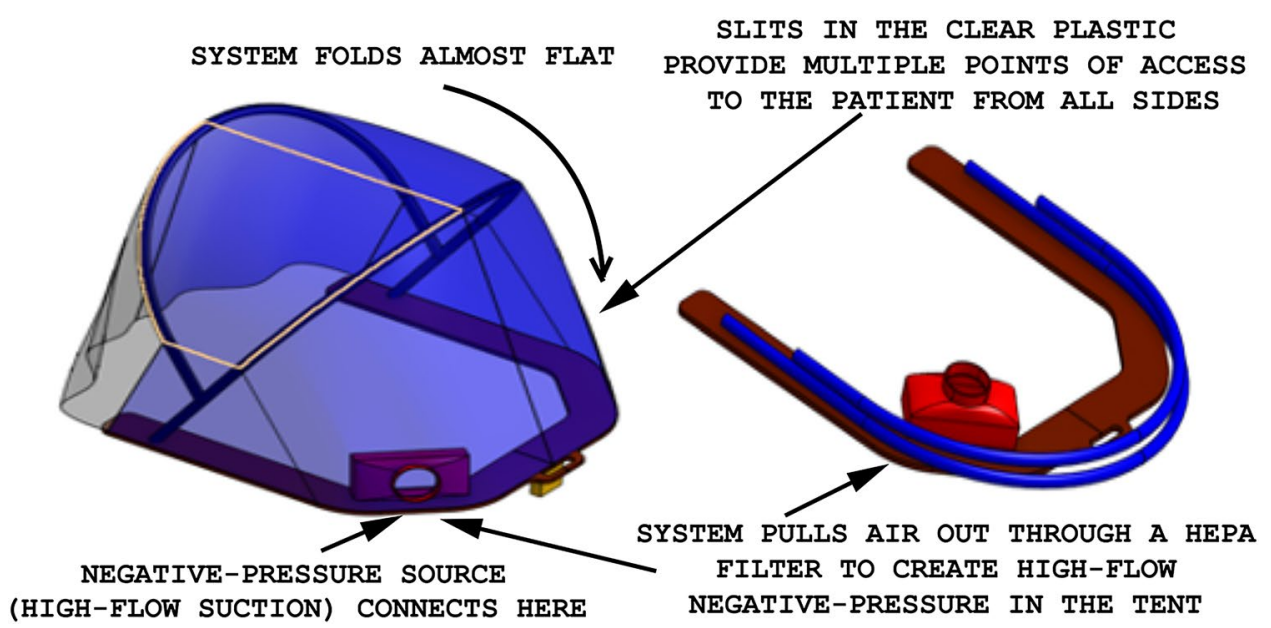

Fig. 1 Diagrammatic representation of the AerosolVE negative-pressure tent

\section{Performance of Tracheostomy}

Indications for and timing of tracheostomy were at the discretion of the primary clinical team. The proceduralists were intensivists with 15 and 5 years' experience performing percutaneous dilatational tracheostomy (PDT). All individuals within the room used appropriate personal protective equipment (PPE), including powered air purifying respirator (PAPR) hoods or N95 masks with face shields. Our technique of PDT using real-time ultrasound guidance has been previously described [25]. The Cook Ciaglia Blue Rhino ${ }^{\circledR}$ (Cook Medical Inc., Bloomington, IN) single-stage dilator PDT kit was used. A Dräger Evita V500 ventilator (Dräger Inc., Lübeck, Germany) was used during the procedures. Patients were deeply sedated with a propofol infusion, pre-treated with fentanyl, and then paralyzed with rocuronium. The tent was first positioned around the patient with the canopy folded back. The skin over the anterior neck was prepared with chlorhexidine, followed by application of sterile drapes and setup of equipment on the sterile field. The canopy was then drawn into position over the sterile field. The proceduralist's arms were introduced through slits in the side of the tent, which were held open by an assistant during hand passage to minimize the risk of contamination of sterile gloves. Bronchoscopy was performed as required by a proceduralist at the head of the bed, through additional slits in the canopy. In accordance with institutional guidelines, the ventilator was placed on standby mode for all anticipated disconnections and disruptions of the closed circuit during the procedure. The endotracheal tube was withdrawn under real-time ultrasound guidance for the first two patients [25], and under bronchoscopic guidance for the third patient.
The remaining steps of PDT were then completed, including local anesthetic, tracheal puncture, passage of the guidewire, confirmation of guidewire position, incision, blunt dissection, dilatation of the stoma, and delivery of the tracheostomy tube [25]. (Figs. 2, 3) Following completion of the procedure, bronchoscopy was performed to confirm good position of the tracheostomy tube. The bronchoscope was also introduced through the tracheostomy tube to confirm good position relative to the carina and perform pulmonary toilet. The tracheostomy tube was secured in place and the tent removed.

\section{Main Outcomes and Measures}

The outcome of interest was successful completion of real-time ultrasound-guided PDT and bronchoscopy within the AerosolVE tent, without complications and without transmission of infection to healthcare staff. Potential complications related to the procedure included, but were not limited to, loss of airway during procedure, cardiac arrest during the procedure, bleeding requiring intervention, stomal or mediastinal infection, posterior wall injury, pneumothorax, pneumomediastinum, nerve injury, sustained hypoxia $\left(\mathrm{SpO}_{2}<90 \%\right.$ for $\left.>1 \mathrm{~min}\right)$ during the procedure, false passage of tube, inability to complete procedure, conversion to surgical tracheostomy, need for revision of tracheostomy, tracheal granuloma, and early dislodgement (within 7 days).

\section{Results}

Three patients underwent PDT with use of the AerosolVE tent. The first patient was a 53-year-old man 


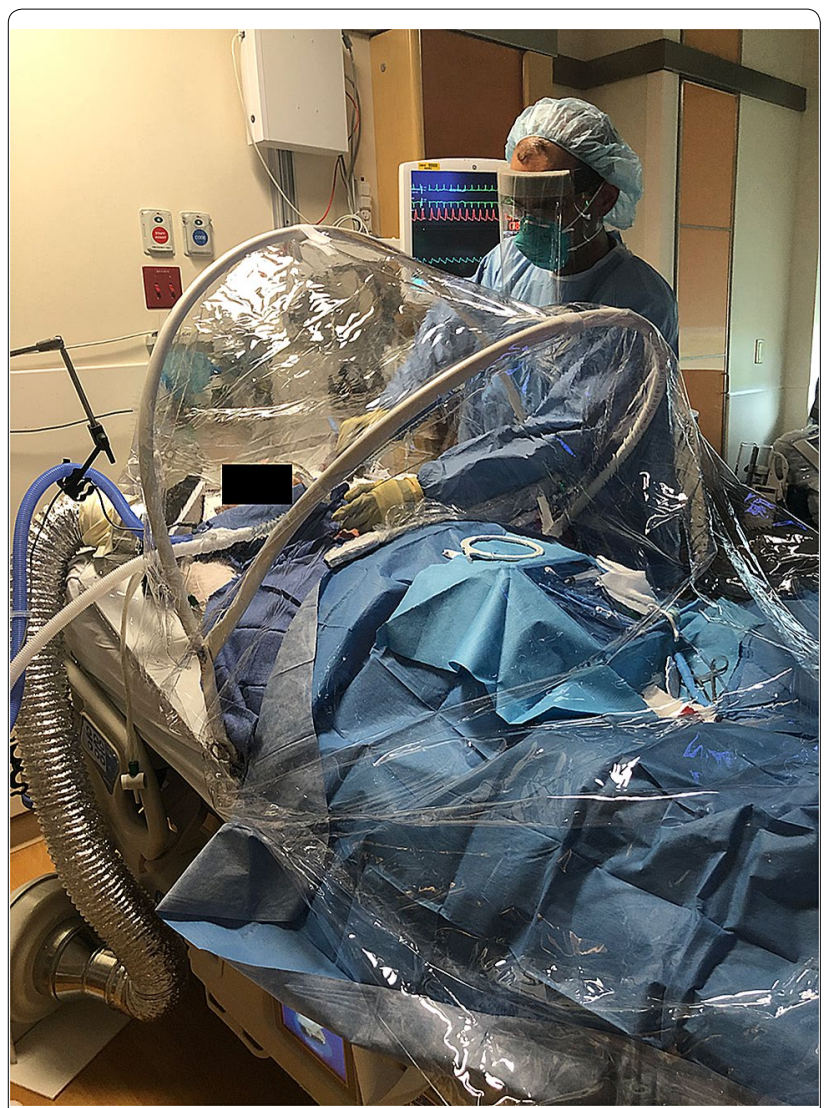

Fig. 2 Performance of tracheostomy within the AerosolVE tentCase 3

with a history of hypertension, with COVID-19-related acute respiratory distress syndrome (ARDS), prolonged unresponsiveness, and multiple bilateral borderzone ischemic strokes intubated 21 days prior to tracheostomy. He was weaned to a $\mathrm{FiO}_{2}$ of $40 \%$ and positive end-expiratory pressure (PEEP) of $8 \mathrm{cmH} 2 \mathrm{O}$, but failed multiple spontaneous breathing trials (SBT) and was insufficiently responsive to reliably protect his airway. $\mathrm{He}$ also remained unresponsive to pain when weaned off all sedation. The second patient was a 53-year-old woman with a history of hypertension, diabetes, coronary artery disease, mitral valve replacement, and COVID-19 who in addition to ARDS also suffered cerebellar hemorrhage, hydrocephalus, and acute severe myopathy. She was intubated 26 days prior to tracheostomy. This patient had been weaned to $\mathrm{FiO}_{2} 40 \%$ and PEEP $10 \mathrm{cmH} 2 \mathrm{O}$ but also failed multiple SBTs and was unable to protect her airway. The third patient was a 69-year-old morbidly obese man with diabetes, hypertension, and coronary artery disease intubated 21 days prior to tracheostomy for COVID-19 associated ARDS. He was on $\mathrm{FiO}_{2} 50 \%$ and PEEP $12 \mathrm{~cm} \mathrm{H}_{2} \mathrm{O}$ and could not be weaned further.
Following initial setup, withdrawal of the endotracheal tube was completed in $<5 \mathrm{~min}$ and the remainder of the procedure under $10 \mathrm{~min}$. Post-procedure bronchoscopy required $<10$ min. Transient hypoxemia $\left(\mathrm{SpO}_{2}<90 \%\right)$ for $<1$ min was observed in the first 2 cases, both times in conjunction with placing the ventilator on standby mode for anticipated disconnections. No sustained hypoxemia or other complications were subsequently observed, and none of the healthcare staff present in the room for either procedure demonstrated signs of infection at the time of this report 4 weeks later.

\section{Discussion}

In this case series, we have demonstrated the feasibility of use of a novel negative-pressure tent during bedside tracheostomy for ventilator-dependent patients with COVID-19. This is the first documented report of PDT performed on COVID-19 patients using a negativepressure device validated to decrease SARS-CoV-2 virussized aerosol, with an air-exchange rate that exceeds the $\mathrm{CDC}$ recommended rate. The device may therefore increase the safety of physicians, nurses, and respiratory therapists involved in these procedures. While use during tracheostomy may represent the most complex application of this device, the device has also been used at our institution for other AGPs such as endotracheal intubation and NIPPV. The device may be particularly valuable when NPRs are unavailable. The device uses low-cost materials and is a significantly cheaper alternative to conversion of existing rooms to NPRs [26]. The portability of the device permits use in varied settings, including the emergency department, intensive care units, operating rooms, other procedural areas, and general care units.

Decreasing risk to healthcare staff during AGPs has implications beyond direct benefit to staff involved. The availability of this device may facilitate tracheostomy performed within a more typical timeframe (7-10 days), without the need for repeated testing or waiting for a more prolonged duration as recommended by some guidelines [6, 7]. Earlier tracheostomy-when clinically appropriate-may improve patient comfort, permit earlier weaning of sedation, and possibly facilitate earlier liberation from the ICU [27]. Of note, the only periods of transient oxygen desaturation during the procedures were during periods of planned discontinuation of mechanical ventilation (standby mode) prior to anticipated circuit breaks to decrease aerosolization, as recommended by current guidelines [6,7]. The very high air-exchange rate within the tent may make such disconnections unnecessary, and thereby increase the safety of the procedure for COVID-19 patients who typically have limited pulmonary reserve and suffer oxygen 


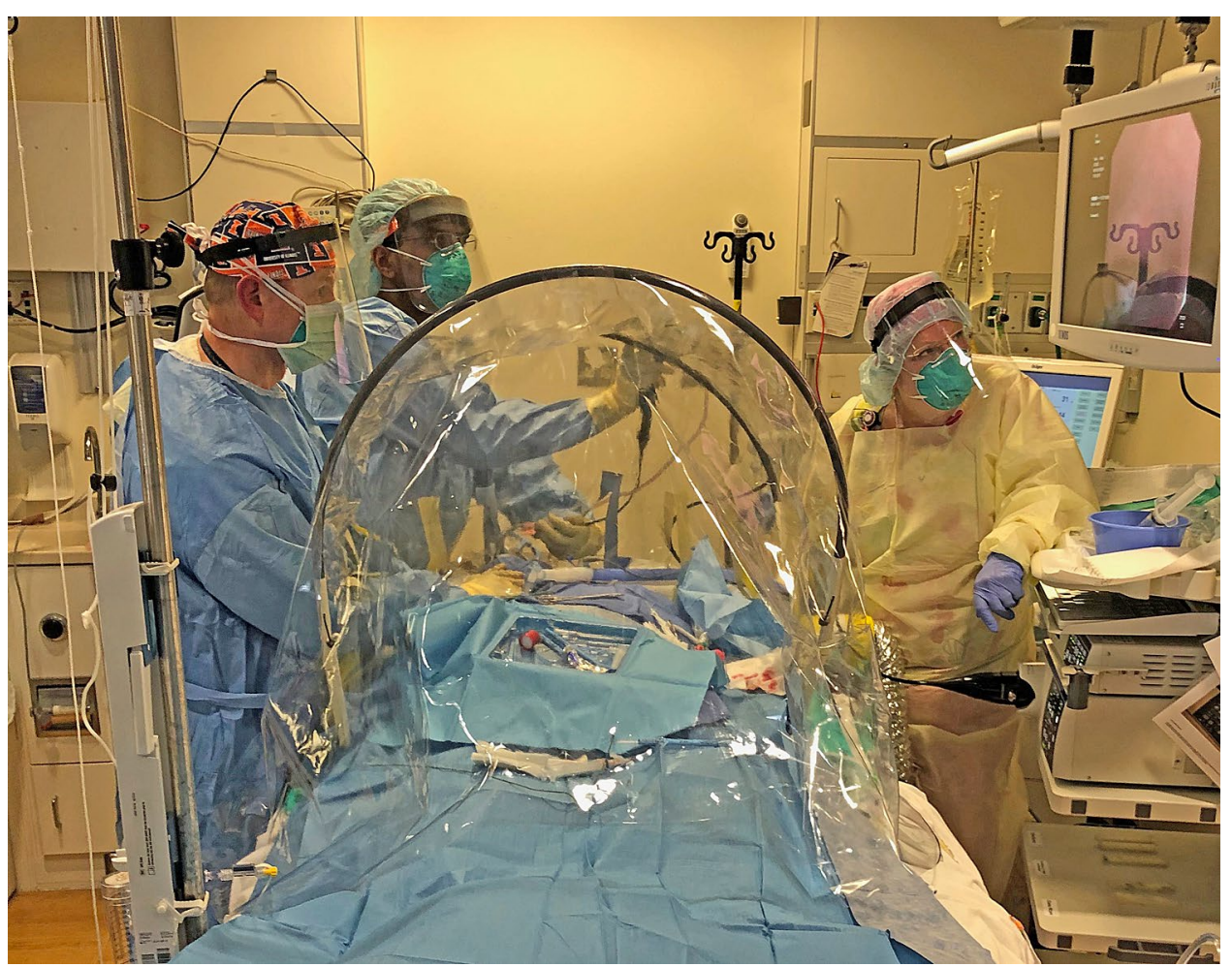

Fig. 3 Bronchoscopy and tracheostomy performed within the AerosolVE tent-Case 2

desaturation with even brief discontinuation of mechanical ventilation.

Several other devices designed to decrease transmission risk during AGPs have been reported during the pandemic, in both the medical literature [8-16] and the lay press [17-19]. The majority of these box, drape, and tent devices do not generate negative pressure in the procedural space. A recent study that utilized a particle counter during simulated AGPs has demonstrated that such devices do not decrease-and may actually increase-the aerosol exposure of operators [20]. Another study has demonstrated that these devices increase failure rates and procedural time during endotracheal intubation [21, 22]. Prior to routine clinical use on COVID-19 patients, devices designed to decrease disease transmission via aerosol must therefore be validated in two ways: 1 ) the ability of the device to greatly decrease the concentration virus-sized (0.06$0.14 \mu \mathrm{m})$ particles in the proximity of the operator and 2) the feasibility of performing the specific procedure in question within the device in the challenging real-world conditions that exist when providing care to COVID-19 patients. Reports from Foster et al. [10] and Bertroche et al. [8] describe custom setups to generate a negativepressure field over the procedural space during tracheostomy, used on one patient each at the time of the reports.
While innovative, these custom-made constructions have not yet been validated with particle counters, and the air-exchange rate has not been reported. Also the need to assemble a field with individual components for each procedure may increase setup time compared to a prefabricated device. Fox et al. have described a negativepressure tent with an air exchange rate of approximately $22 / \mathrm{h}$; however, this is substantially lower than the 600 exchanges/h documented with AerosolVE [11]. Also this tent may not be tailored to sterile procedures, and clinical use of this device has not yet been reported [11]. Convissar and colleagues have described the use of a negativepressure system for AGPs in the operating room that utilizes a Stryker Neptune ${ }^{\text {TM }}$ (Stryker Corporation, Kalamazoo, MI) high-power suction system [9]. However, this system is relatively small and has not yet demonstrated feasibility of use with tracheostomy. Adverse events have occurred when the Stryker ${ }^{\mathrm{TM}}$ system, which is compatible with a variety of tubing used in routine clinical care, was inadvertently connected to chest tubes [9].

This case-series only demonstrates feasibility. Larger studies are necessary to optimally demonstrate the safety and efficacy of this device. The most significant limitation observed was the risk of glove contamination within the tent, and the relatively confined procedural space, although neither proceduralist reported 
such contamination during the 3 cases in this report. The consequences of inadvertent contamination may be lower in procedures performed on a non-sterile region such as the airway. A newer version of the tent is larger and provides greater procedural space in addition to a higher air-flow rate. It should also be noted that tracheostomy is an elective procedure that allows time to position and setup a negative-pressure device. Emergency AGPs such as endotracheal intubation performed with any negative-pressure device should likely be simulated on mannequins before they are performed on COVID-19 patients.

We conclude that it is feasible to perform bedside tracheostomy on COVID-19 patients while using the AerosolVE negative-pressure tent. This is a promising low-cost device to decrease risk to healthcare providers during AGPs.

\begin{abstract}
Author details
1 Department of Neurosurgery, University of Michigan, 1500 E. Medical Center Drive, 3552 Taubman Health Care Center, SPC 5338, Ann Arbor, Ml 48109, USA. ${ }^{2}$ Department of Neurology, University of Michigan, Ann Arbor, MI, USA. ${ }^{3}$ Michigan Center for Integrative Research in Critical Care, University of Michigan, Ann Arbor, MI, USA.
\end{abstract}

\section{Acknowledgements}

We would like to acknowledge Nathan L. Haas, MD, Henrique Alencastro Puls, $M D$, Kevin R. Ward, MD, and Benjamin S. Bassin, MD, faculty in the department of emergency medicine and members of the Michigan Center for Integrative Research in Critical Care at the University of Michigan who helped design the tent and facilitated availability for clinical use. We would also like to acknowledge Sridhar Kota, PhD, professor of mechanical engineering at the University of Michigan and FlexSys Inc. of Ann Arbor, Ml, who collaborated in the design and manufactured the AerosolVE tent. We would also like to thank Shahid N. Ahmad, MBBS, who assisted with the performance of tracheostomy.

\section{Author Contributions}

VR conceived of the study, designed the study, performed data collection, drafted the manuscript and edited the manuscript. CAW helped conceive the study, performed data collection and edited the manuscript. All authors have read and approved the final version of the manusript.

\section{Source of support}

None.

\section{Conflicts of interest}

The authors declare that they have no conflict of interest.

\section{Ethical approval/Informed consent}

The University of Michigan Institutional Review Board (IRB) reviewed the study and waived the need for approval. Clinical use of the tent during the pandemic was under an institutional innovative clinical care protocol. Informed consent was obtained from legally authorized representatives for use of the tent, as well as acquisition and publication of photographic images and video.

\section{Publisher's Note}

Springer Nature remains neutral with regard to jurisdictional claims in published maps and institutional affiliations.

Received: 16 June 2020 Accepted: 25 July 2020

Published online: 7 August 2020

\section{References}

1. Meng L, Qiu H, Wan L, et al. Intubation and ventilation amid the COVID19 Outbreak: Wuhan's Experience. Anesthesiology. 2020;132(6):1317-32.

2. Zhou F, Yu T, Du R, et al. Clinical course and risk factors for mortality of adult inpatients with COVID-19 in Wuhan, China: a retrospective cohort study. Lancet. 2020;395(10229):1054-62.

3. Chou R, Dana T, Buckley DI, et al. Epidemiology of and risk factors for coronavirus infection in Health Care Workers: a living rapid review. Ann Intern Med. 2020. https://doi.org/10.7326/M20-1632.

4. Heinzerling A, Stuckey MJ, Scheuer T, et al. Transmission of COVID-19 to health care personnel during exposures to a hospitalized patient Solano County, California, February 2020. MMWR Morb Mortal Wkly Rep. 2020;69(15):472-6.

5. Tran K, Cimon K, Severn M, Pessoa-Silva CL, Conly J. Aerosol generating procedures and risk of transmission of acute respiratory infections to healthcare workers: a systematic review. PLoS ONE. 2012;7(4):e35797.

6. Michetti CP, Burlew CC, Bulger EM, et al. Performing tracheostomy during the Covid-19 pandemic: guidance and recommendations from the Critical Care and Acute Care Surgery Committees of the American Association for the Surgery of Trauma. Trauma Surg Acute Care Open. 2020;5(1):e000482.

7. Parker NP, Schiff BA, Fritz MA, et al. Tracheotomy recommendations during the COVID-19 Pandemic-Airway and Swallowing Committee of the American Academy of Otolaryngology-Head and Neck Surgery. 2020 April 22020 [cited 2020 May 15 2020]; https://www.entnet.org/content/ tracheotomy-recommendations-during-covid-19-pandemic.

8. Bertroche JT, Pipkorn P, Zolkind P, Buchman CA, Zevallos JP. NegativePressure Aerosol Cover for COVID-19 Tracheostomy. JAMA Otolaryngol Head Neck Surg. 2020;146:672.

9. Convissar D, Chang CY, Choi WE, Chang MG, Bittner EA. The vacuum assisted negative pressure isolation hood (VANISH) system: novel application of the stryker neptune suction machine to create COVID-19 negative pressure isolation environments. Cureus. 2020;12(5):e8126.

10. Foster P, Cheung T, Craft P, et al. Novel approach to reduce transmission of COVID-19 during tracheostomy. J Am Coll Surg. 2020;230(6):1102-4.

11. Fox $T H$, Silverblatt M, Lacour A, deBoisblanc BP. Negative pressure tent to reduce exposure of health care workers to SARS CoV-2 during aerosol generating respiratory therapies. Chest. 2020. https://doi.org/10.1016/j. chest.2020.04.070.

12. Au Yong $P S$, Chen $X$. Reducing droplet spread during airway manipulation: lessons from the COVID-19 pandemic in Singapore. Br J Anaesth. 2020;125(1):e176-e8.

13. Adir Y, Segol O, Kompaniets D, et al. COVID-19: minimising risk to healthcare workers during aerosol-producing respiratory therapy using an innovative constant flow canopy. Eur Respir J 2020;55(5):2001017. https:// doi.org/10.1183/13993003.01017-2020.

14. Seger $C D$, Wang $L$, Dong $X$, et al. A novel negative pressure isolation device for aerosol transmissible COVID-19. Anesth Analg 2020. https:// doi.org/10.1213/ANE.0000000000005052.

15. Canelli R, Connor CW, Gonzalez M, Nozari A, Ortega R. Barrier enclosure during endotracheal intubation. N Engl J Med. 2020;382(20):1957-8.

16. Hill E, Crockett C, Circh RW, Lansville F, Stahel PF. Introducing the "Corona Curtain": an innovative technique to prevent airborne COVID-19 exposure during emergent intubations. Patient Saf Surg. 2020;14:22.

17. Everington K. Taiwan News. Taiwanese doctor invents device to protect US doctors against coronavirus. 2020 [cited 2020 March 23]; https://www. taiwannews.com.tw/en/news/3902435.

18. Tercek K 14NEWS. Keusch Glass Inc. creates intubation boxes for medical personnel. 2020 [cited 2020 April 1]; https://www.14new s.com/2020/04/02/keusch-glass-inc-creates-intubation-boxes-medic al-personnel/.

19. Colasimone D. ABC News. Australian doctors design and make life-saving equipment needed for coronavirus pandemic. 2020 [cited 2020 April 5]; https://www.abc.net.au/news/2020-04-06/doctors-designing-medic al-equipment-to-face-coronavirus-covid-19/12120588.

20. Simpson JP, Wong DN, Verco L, et al. Measurement of airborne particle exposure during simulated tracheal intubation using various proposed aerosol containment devices during the COVID-19 pandemic. Anaesthesia 2020. https://doi.org/10.1111/anae.15188.

21. Ortega R, Nozari A, Canelli R. More on barrier enclosure during endotracheal intubation. Reply N Engl J Med. 2020;382(21):e69. 
22. Begley JL, Lavery KE, Nickson CP, Brewster DJ. The aerosol box for intubation in coronavirus disease 2019 patients: an in-situ simulation crossover study. Anaesthesia. 2020;75(8):1014-21.

23. Sehulster L, Chinn RY. CdcHicpac Guidelines for environmental infection control in health-care facilities. Recommendations of CDC and the Healthcare Infection Control Practices Advisory Committee (HICPAC). MMWR Recomm Rep. 2003;52:1-42.

24. Bassin BS, Haas NL, Puls HA, et al. Rapid development of a portable negative pressure procedural tent. Int J Tuberculosis Lung Dis 2020;24(7):74043. https://doi.org/10.5588/ijtld.20.0317.
25. Rajajee $V$, Williamson CA, West BT. Impact of real-time ultrasound guidance on complications of percutaneous dilatational tracheostomy: a propensity score analysis. Crit Care. 2015;19:198.

26. Dowdy DW, Maters A, Parrish N, Beyrer C, Dorman SE. Cost-effectiveness analysis of the gen-probe amplified mycobacterium tuberculosis direct test as used routinely on smear-positive respiratory specimens. J Clin Microbiol. 2003;41(3):948-53.

27. Terragni PP, Antonelli M, Fumagalli R, et al. Early vs late tracheotomy for prevention of pneumonia in mechanically ventilated adult ICU patients: a randomized controlled trial. JAMA. 2010;303(15):1483-9. 